\title{
Dengue induces platelet activation, mitochondrial dysfunction and cell death through mechanisms that involve DC-SIGN and caspases
}

E. D. HOTTZ, ${ }^{*} \dagger M$. F. OLIVEIRA, $+P . C . G . N U N E S, \S R . M . R . N O G U E I R A, \S R$. VALLS-DE-SOUZA, $\dagger$ A. T. DA POIAN, + A. S. WEYRICH, ${ }^{* *}$ G. A. ZIMMERMAN, ${ }^{*} *$ P. T. BOZZA*1 and F. A. BOZZA $\dagger^{1}$ * Laboratório de Immunofarmacologia, Instituto Oswaldo Cruz, Fundação Oswaldo Cruz; †Instituto de Pesquisa Clinica Evandro Chagas, Fundação Oswaldo Cruz; łInstituto de Bioquímica Médica, Universidade Federal do Rio de Janeiro; §Laboratório de Flavivirus, Instituto Oswaldo Cruz, Fundação Oswaldo Cruz, Rio de Janeiro, Brazil; @The Molecular Medicine Program, University of Utah; and **Department of Medicine, University of Utah, Salt Lake City, UT, USA

To cite this article: Hottz ED, Oliveira MF, Nunes PCG, Nogueira RMR, Valls-de-Souza R, Da Poian AT, Weyrich AS, Zimmerman GA, Bozza PT, Bozza FA. Dengue induces platelet activation, mitochondrial dysfunction and cell death through mechanisms that involve DC-SIGN and caspases. J Thromb Haemost 2013; 11: 951-62.

Summary. Background: Worldwide, dengue is the most prevalent human arbovirus disease. Dengue infection may cause a range of clinical manifestations from self-limiting febrile illness through to a life-threatening syndrome accompanied by both bleeding and shock. Thrombocytopenia is frequently observed in mild and severe disease; however, the mechanisms involved in DENV-induced platelet activation and thrombocytopenia are incompletely understood. Patients and methods: Freshly isolated platelets from patients with dengue were evaluated for markers of activation, mitochondrial alteration and activation of cell death pathways. In parallel, we examined direct DENV-induced activation and apoptosis of platelets obtained from healthy subjects. Results: We found that platelets from DENVinfected patients exhibited increased activation by comparison to control subjects. Moreover, platelets from DENVinfected patients exhibited classic signs of the intrinsic pathway of apoptosis that include increased surface phosphatidylserine exposure, mitochondrial depolarization and

Correspondence: Fernando A. Bozza, Instituto de Pesquisa Clinica Evandro Chagas, Fundação Oswaldo Cruz, Rio de Janeiro, Brazil. Tel.: 5521 25621311; fax: 552125903545.

E-mail: fernando.bozza@ipec.fiocruz.br or bozza.fernando@gmail. com

\section{and}

Patricia T. Bozza, Laboratório de Immunofarmacologia, Instituto Oswaldo Cruz, Fundação Oswaldo Cruz, Rio de Janeiro, Brazil. Tel.: 5521 25621767; fax: 552125903545.

E-mail: pbozza@ioc.fiocruz.br

${ }^{1}$ The senior authors contributed equally.

Received 11 December 2012, accepted 19 February 2013 activation of caspase- 9 and -3 . Indeed, thrombocytopenia was shown to strongly associate with enhanced platelet activation and cell death in DENV-infected patients. Platelet activation, mitochondrial dysfunction and caspasedependent phosphatidylserine exposure on platelets were also observed when platelets from healthy subjects were directly exposed to DENV in vitro. DENV-induced platelet activation was shown to occur through mechanisms largely dependent on DC-SIGN. Conclusions: : Together our results demonstrate that platelets from patients with dengue present signs of activation, mitochondrial dysfunction and activation of the apoptosis caspase cascade, which may contribute to the development of thrombocytopenia in patients with dengue. Our results also suggest the involvement of DC-SIGN as a critical receptor in DENV-dependent platelet activation.

Keywords: apoptosis, DC-SIGN, dengue, mitochondrial dysfunction, platelet activation, thrombocytopenia.

\section{Introduction}

Dengue is an infectious disease caused by the dengue virus (DENV). Over 2.5 billion people live in high-risk transmission areas and dengue has emerged as a problem in the Southern United States [1]. It is estimated that over 50 million individuals are annually exposed to DENV [2]. The natural history of dengue is a self-limiting fever followed by a critical phase of defervescence, in which the patient may improve or progress to severe dengue that is associated with life-threatening increases in vascular permeability, hypovolemia, hypotension and shock [2,3]. Thrombocytopenia is commonly observed in both mild and severe dengue syndromes and correlates with the clinical outcome [2-6]. 
The potential mechanisms inciting DENV-associated thrombocytopenia were recently reviewed [7]. These include impaired thrombopoiesis [8] and/or peripheral platelet destruction. The latter may involve antibodyinduced platelet clearance [9], enhanced interactions of platelets with leukocytes or endothelium $[10,11]$ and activation of platelets as they contact the DENV $[12,13]$. Recent studies suggest that DENV is able to interact with platelets and, in turn, induce ultrastructural changes [12]. Platelets also become activated when they are exposed to DENV-infected endothelial cells [10] and increased numbers of platelet-monocyte aggregates have been observed in dengue patients [11]. These previous studies indicate that platelets can become activated during dengue infection, but the mechanisms underlying this process and its clinical consequences remain unknown.

Mitochondria are known to regulate apoptotic pathways in activated platelets [14-16]. Therefore, we examined platelet function in patients with dengue, focusing on how dengue influences mitochondrial function and apoptosis. We found that platelet activation is significantly increased in dengue-infected patients, especially in individuals with thrombocytopenia. Platelets from patients with dengue also had impaired mitochondrial function and exhibited signs of apoptosis pathway activation. Consistent with these findings, in vitro DENV infection induces similar responses of activation, mitochondrial dysfunction and apoptosis through mechanisms involving Dendritic Cell-Specific Intercellular adhesion molecule-3-Grabbing Non-integrin (DC-SIGN). Our results suggest that DENV-induced platelet activation and apoptosis occurs though DC-SIGN-dependent mechanisms and may influence the development of thrombocytopenia in dengue.

\section{Patients and methods}

\section{Human subjects}

We prospectively followed a cohort of 39 serologically/ molecularly confirmed DENV-infected patients from the Instituto Pesquisas Clínicas Evandro Chagas, FIOCRUZ, Rio de Janeiro, Brazil, whose characteristics are presented in Table 1. Peripheral vein blood samples were obtained at febrile $(n=26)$, defervescence $(n=26)$ and convalescence $(n=13)$ phases of infection. The mean day of sample collection after the onset of illness was $4.5 \pm 1.6$ in febrile, $7.5 \pm 2.4$ in defervescence and $25.3 \pm 12.9$ in convalescence. Primary and secondary infections were distinguished using the $\operatorname{IgM} / \operatorname{IgG}$ antibody ratio as previously described $[2,17]$. For virus typing and quantification, viral RNA was extracted (QIAamp Viral RNA; Qiagen, Valencia, CA, USA) from plasma samples and processed as previously described $[18,19]$.

Peripheral vein blood was also collected from 30 agedmatched healthy subjects and 13 patients with non-dengue febrile illness (NDFI). The disease etiologies of the NDFI
Table 1 Characteristics of dengue-infected patients

\begin{tabular}{|c|c|c|}
\hline & Control (30) & Dengue (39) \\
\hline Age, years & $27(26-35)$ & $37(26-46)$ \\
\hline Gender, male & $12(40 \%)$ & $22(56.4 \%)$ \\
\hline Platelet count, $\times 1000 \mathrm{~mm}^{-3}$ & - & $107(49-145)$ \\
\hline Hematocrit, \% & - & $43.5(40.7-45.3$ \\
\hline Albumin, $\mathrm{g} \mathrm{dL}^{-1}$ & - & $3.6(3.1-3.9)$ \\
\hline $\mathrm{TGO} / \mathrm{AST}, \mathrm{IU} \mathrm{L}^{-1}$ & - & $66(35-115)$ \\
\hline $\mathrm{TGP} / \mathrm{ALT}, \mathrm{IU} \mathrm{L}^{-1}$ & - & $69(46-102)$ \\
\hline Haemorrhagic manifestations* & - & $11(28.2 \%)$ \\
\hline Venous hydration & - & $16(41 \%)$ \\
\hline Secondary infection & - & $24(61 \%)$ \\
\hline Mild dengue & - & $20(51.3 \%)$ \\
\hline Mild dengue with warning signs $\dagger$ & - & $15(38.5 \%)$ \\
\hline Severe dengue & - & $4(10.2 \%)$ \\
\hline PCR positive & - & 29 \\
\hline DENV-1 & - & $27(93 \%)$ \\
\hline DENV-2 & - & $02(7 \%)$ \\
\hline Viremia, $\times 10^{5}$ copies $\mathrm{mL}^{-1}$ & - & $1.5 \pm 3.4$ \\
\hline
\end{tabular}

Data are expressed as median (interquartile range) or number (\%).

* Gingival bleed, vaginal bleed, gastrointestinal bleed, petechiae and exanthema.

$\dagger$ Abdominal pain or tenderness, persistent vomiting, clinical fluid accumulation, mucosal bleed, and/or increased hematocrit concurrent with rapid decrease in platelet count. According to WHO guidelines [2].

†Severe plasma leakage, fluid accumulation, ascites and/or massive bleeding. According to WHO guidelines [2].

patients are listed in Table S1. Written informed consent was obtained from all participants prior to any studyrelated procedure. The study protocol was approved by the Institutional Review Board (IPEC\#016/2010).

\section{Platelet isolation}

Platelets were isolated as previously described [20] and resuspended in medium 199 at $37^{\circ} \mathrm{C}$. The purity of the platelet preparations (>99\% of $\mathrm{CD} 41^{+}$events) was confirmed by flow cytometry.

\section{Flow cytometric analysis}

Platelets were resuspended in modified Tyrode's $(137 \mathrm{~mm}$ $\mathrm{NaCl}, 2.68 \mathrm{~mm}$ KCI, $5 \mathrm{~mm}$ HEPES, $1 \mathrm{~mm} \mathrm{MgCl}_{2}$, 11.9 mм $\mathrm{NaHCO}_{3}, 0.42 \mathrm{~mm} \mathrm{NaH}_{2} \mathrm{PO}_{4}, 4.7 \mathrm{~mm}$ glucose; $\mathrm{pH}$ 7.4). P-selectin (CD62-P) surface expression was determined using PE- or FITC-conjugated anti-human CD62P (BD Pharmingen, San Jose, CA, USA) $\left(0.25 \mu \mathrm{g} \mathrm{mL}{ }^{-1}\right.$, $30 \mathrm{~min}$ ); DC-SIGN surface expression was determined by incubating platelets with biotin-conjugated antibody against DC-SIGN (eBioscience, San Diego, CA, USA; eBh209) (1.5 $\left.\mu \mathrm{g} \mathrm{mL}^{-1}, 20 \mathrm{~min}\right)$ and PECy5-conjugated streptavidin $\left(0.2 \mu \mathrm{g} \mathrm{mL}^{-1}, 20 \mathrm{~min}\right)$; mitochondrial membrane potential $\left(\Delta \Psi_{\mathrm{m}}\right)$ was measured using the probe, tetramethylrhodamine ethyl ester (TMRE, Fluka Analytical, San Diego, CA, USA) (100 nм, $10 \mathrm{~min})$, mitochondrialderived reactive oxygen species $\left(\operatorname{ROS}_{\mathrm{m}}\right)$ were detected 
using MitoSoxRed (Invitrogen, Carlsbad, CA, USA) ( $2.5 \mu \mathrm{M}, 10 \mathrm{~min}$ ); active caspase-9 was determined using green FAM-LEDH-FMK, FLICA (Immunochemistry Technologies, Bloomington, MN, USA); and phosphatidylserine exposure was stained with FITC-conjugated Annexin V (BD Pharmingen). A minimum of 10000 gated events were acquired using a FACScalibur flow cytometer (BD Bioscience, San Diego, CA, USA).

\section{Assessment of mitochondrial function}

TMRE and MitoSoxRed were used to measure $\Delta \Psi_{\mathrm{m}}$ and $\mathrm{ROS}_{\mathrm{m}}$, respectively. To control the mitochondrial specificity of the probes, platelets were treated with the proton ionophore FCCP $(0.5 \mu \mathrm{M}) 15 \mathrm{~min}$ before labeling. To assess mitochondrial permeability, platelets were treated with the $F_{1} F_{0}$-ATP-synthase inhibitor Oligomycin $\left(1 \mu \mathrm{g} \mathrm{mL}^{-1}\right) 15 \mathrm{~min}$ before $\Delta \Psi_{\mathrm{m}}$ labeling. Mitochondrial content was determined measuring citrate-synthase activity in platelet protein extracts $(10 \mu \mathrm{g})$ by $412 \mathrm{~nm}$ spectrophotometric detection of CoA-TNB2, which formed with citrate as DTNB, acetyl-CoA and oxaloacetate interacted with one another.

\section{Western blotting}

Western blotting was performed as previously described [21]. The primary antibodies used were mouse anti-human caspase-9 and rabbit anti-human caspase-3 (Cell Signaling Technology, Danvers, MA, USA).

\section{Virus preparation}

DENV serotype 2 strain 16881 was propagated in C6/36 Aedes albopictus mosquito cells and titrated by plaque assay on BHK cells [22]. The amount of infectious particles was expressed as plaque forming units (PFU) per $\mathrm{mL}$. Supernatants from uninfected cell cultures (mock) were produced using the same conditions.

\section{In vitro platelet stimulation}

Platelets from healthy volunteers were incubated with thrombin (T1063; Sigma, St. Louis, MO, USA) $\left(0.1 \mathrm{U} \mathrm{mL}^{-1}\right)$ or with DENV-2 (1 PFU per platelet) at $37{ }^{\circ} \mathrm{C}$ for the indicated times. In selected experiments platelets were pre-incubated for $30 \mathrm{~min}$ with neutralizing antibodies against DC-SIGN (120507; R\&D Systems, Minneapolis, MN, USA) $(25 \mu \mathrm{g}$ $\mathrm{mL}^{-1}$ ), the integrin $\alpha_{\mathrm{V}}$ subunit, or an isotype-matched antibody; or with the pan-caspase inhibitor ZVAD-fmk (BioVision, Milpitas, CA, USA) $\left(20 \mu \mathrm{g} \mathrm{mL} \mathrm{m}^{-1}\right)$.

\section{Statistical analysis}

Statistics were performed using GraphPad Prism 5.0 software (GraphPad Software, San Diego, CA, USA). One- way analysis of variance (ANOVA) was used to determine differences. Bonferroni's post-hoc test was employed to identify the location of each difference between groups. The paired two-tail $t$-test was used to compare stimulated and unstimulated platelets from the same donor. Correlations were assessed using Pearson's test.

\section{Results}

\section{Platelet activation in patients with dengue}

The intensity of surface P-selectin expression (mean fluorescence intensity, MFI) on platelets was significantly $(P<0.05)$ higher in samples from patients with dengue during the febrile $(51.5 \pm 24.3 \mathrm{MFI})(41.0 \pm 22.0 \mathrm{MFI})$ phases compared with the convalescence $(22.9 \pm 6.3 \mathrm{MFI})$ phase or healthy volunteers $(16.9 \pm 4.3 \mathrm{MFI})$. P-selectin expression was also higher on platelets isolated from febrile and defervescence DENV-infected patients compared with
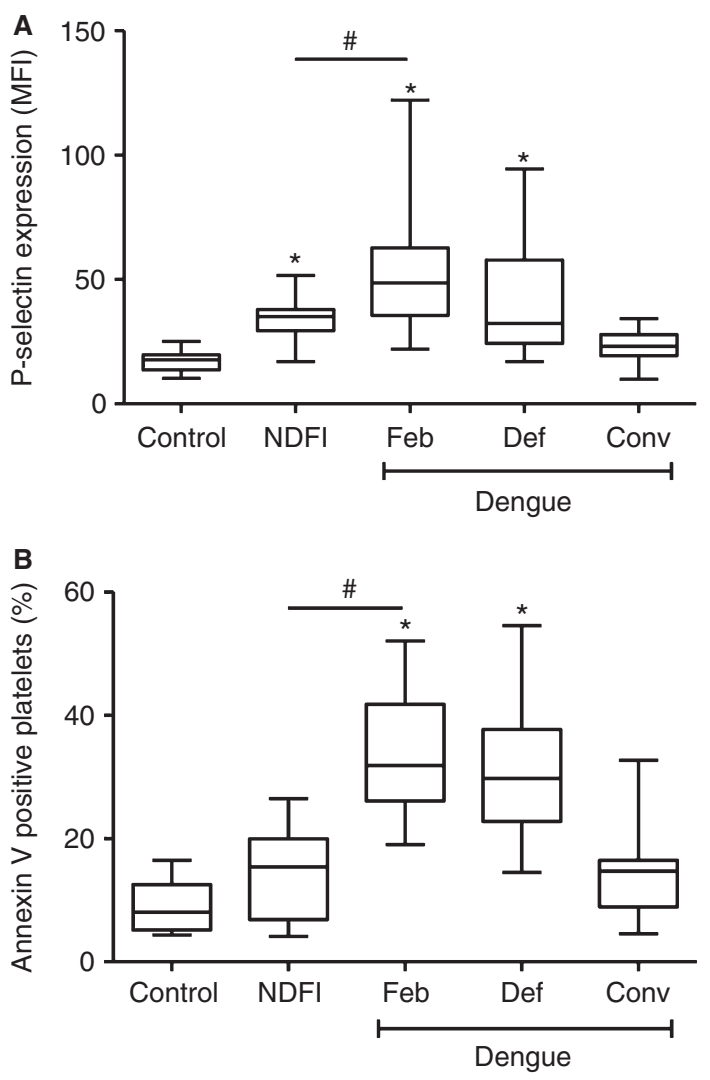

Fig. 1. Platelet activation is increased during dengue illness. The mean fluorescence intensity (MFI) of P-selectin expression (A) and the percentage of annexin V-binding platelets (B) in platelets freshly isolated from healthy subjects (control), patients with non-dengue febrile illness (NDFI), and dengue-infected patients in febrile (Feb), defervescence (Def), and convalescence (Conv) phases. Boxes indicate median and interquartile ranges and whiskers indicate 5-95 percentile. $* P<0.05$ vs. control; $\# P<0.05$ vs. non-dengue febrile illness (NDFI). 
NDFI $(33.8 \pm 8.7 \mathrm{MFI})$ even although surface P-selectin expression was greater in NDFI compared with healthy subjects (Fig. 1A).

Phosphatidylserine exposure on platelets (percentage of positive platelets) was similarly increased $(P<0.01)$ in patients in febrile $(34.4 \pm 10.4 \%)$ and defervescence $(31.6 \pm 10.7 \%)$ dengue phases vs. patients in the convalescence phase $(14.1 \pm 7.3 \%)$, healthy volunteers $(9.1 \pm 4.2 \%)$, or NDFI $(14.3 \pm 7.6 \%)$ (Fig. 1B).

\section{Platelet activation is associated with thrombocytopenia during dengue disease}

Using platelet counts determined on the day of sample collection, patients were classified as thrombocytopenic (TCP) $\quad\left(<150000 \mathrm{~mm}^{-3}\right)$ or non-thrombocytopenic (NTCP). Based on this grouping, 14 patients in the febrile phase were TCP whereas 12 were NTCP. The breakdown was similar in dengue patients in the defervescence phase (14 TCP and 10 NTCP patients). P-selectin surface expression was higher in TCP dengue patients $(61.5 \pm 27.5$ MFI for febrile and $49.3 \pm 25.2$ MFI for defervescence) vs. NTPC $(39.8 \pm 13.0$ for febrile and $29.8 \pm 6.6 \mathrm{MFI}$ for defervescence) (Fig. 2A).

Moreover, we found that platelet counts in DENVinfected patients inversely correlated with surface P-selectin expression and phosphatidylserine exposure (Fig. 2B, C). This relationship between thrombocytopenia and platelet activation was similarly observed during the course of the disease (Fig. 2D).

\section{Mitochondrial dysfunction in platelets from patients with dengue}

Mitochondria are important regulators of the intrinsic pathways of apoptosis [23]. They also regulate activation responses and procoagulant activity in platelets [24], and loss of $\Delta \Psi_{\mathrm{m}}$ occurs in platelets that become apoptotic after activation $[14,15]$ or aging during storage [16,25]. As shown in Fig. 3A, the proton ionophore FCCP significantly reduced $\Delta \Psi_{\mathrm{m}}$ in platelets isolated from healthy subjects or DENV-infected patients. However, basal $\Delta \Psi_{\mathrm{m}}$ was significantly $(P<0.01)$ reduced in platelets isolated from dengue-infected patients in febrile $(14.6 \pm 3.5 \mathrm{MFI})$ and defervescence $(14.6 \pm 4.1 \mathrm{MFI})$ compared with dengue patients in the convalescence $(21.8 \pm 3.6 \mathrm{MFI})$, NDFI $(20.5 \pm 4.6 \mathrm{MFI})$, or healthy volunteers $(23.2 \pm 4.6 \mathrm{MFI})$ (Fig. 3B). As expected, $\Delta \Psi_{\mathrm{m}}$ was significantly increased when platelets from healthy subjects were incubated with oligomycin, which inhibits mitochondrial $\mathrm{H}^{+}$-ATP synthase. This increase, however, was not observed when platelets from dengue patients were exposed to oligomycin (Fig. 3C).

Next, we assessed citrate-synthase activity in platelets and found no differences between dengue patients and controls (Fig. 3D). These results indicate that perturba- tions in $\Delta \Psi_{\mathrm{m}}$ are result of increased mitochondrial permeability and not a reduction in mitochondrial content.

As shown in Fig. 3E,F, platelets from DENV-infected patients constitutively generate $\mathrm{ROS}_{\mathrm{m}}$. Treatment of the platelets with FCCP confirmed that ROS were derived from mitochondria (Fig. 3E). Basal production of $\mathrm{ROS}_{\mathrm{m}}$ was significantly $(P<0.05)$ higher in platelets from dengue patients at both febrile $(14.2 \pm 3.8 \mathrm{MFI})$ and defervescence $(11.8 \pm 2.7 \mathrm{MFI})$ phases compared with dengue patients at the convalescence $(8.4 \pm 0.9 \mathrm{MFI})$, NFDI $(9.7 \pm 2.2$ MFI) and healthy subjects ( $8.4 \pm 1.3 \mathrm{MFI})$ (Fig. 3F).

\section{Platelet apoptosis in patients with dengue}

Increased phosphatidylserine exposure and mitochondrial depolarization suggest that apoptotic pathways are more active in platelets from DENV-infected patients. Consistent with this possibility, we found that phosphatidylserine exposure negatively correlated with $\Delta \Psi_{\mathrm{m}}$ in platelets from dengue patients (Fig. 4A). To assess activation of apoptosis pathways in more depth, we measured activation of caspase- 9 and -3 in platelets during dengue illness. Caspase-9 activation was significantly $(P<0.01)$ higher in platelets isolated from febrile $(15.3 \pm 3.2 \mathrm{MFI})$ and defervescence dengue patients compared with healthy subjects $(8.5 \pm 1.7 \mathrm{MFI})$, dengue patients in convalescence $(8.8 \pm 2.5 \mathrm{MFI})$ and NDFI $(11.3 \pm 2.3 \mathrm{MFI})$ (Fig. 4B). Higher amounts of cleaved caspase-9 and -3 were also observed by Western blot in DENV-infected patients compared with healthy volunteers (Fig. 4C).

\section{DENV-2 induces activation, mitochondrial dysfunction and apoptosis in platelets}

Next, we determined whether DENV-2 directly activates platelets yielding similar responses to those of platelets from dengue-infected patients. Platelets from healthy donors were incubated with DENV-2 or mock for 1 , $30 \mathrm{~min}, 1.5,3$ and $6 \mathrm{~h}$. Incubation of platelets with DENV-2 significantly increased surface P-selectin expression at $6 \mathrm{~h}$ compared with mock $(55.2 \pm 5.7$ vs. $30.7 \pm 12.4$ MFI; $n=4, P=0.019$ ). The kinetics of P-selectin presentation on the surface of platelets exposed to DENV-2 was distinct from that of thrombin stimulation, suggesting that DENV activates platelets through different mechanisms (Fig. 5A).

Six hours exposure to purified DENV-2 similarly activated platelets (Fig. 5B). Virus purification was obtained using a Centricon YM-100 Centrifugal Filter (Merck Millipore, Billerica, MA, USA) and platelets from healthy subjects were exposed separately to the filtrate or to the retentate (purified virus) resuspended in medium. Exposure of platelets to purified DENV-2 significantly increased surface P-selectin expression compared with the virus filtrate or to mock sample processed in parallel (purified DENV-2, $49.6 \pm 12.5$ MFI vs. $23.6 \pm 14.5 \mathrm{MFI}$ 

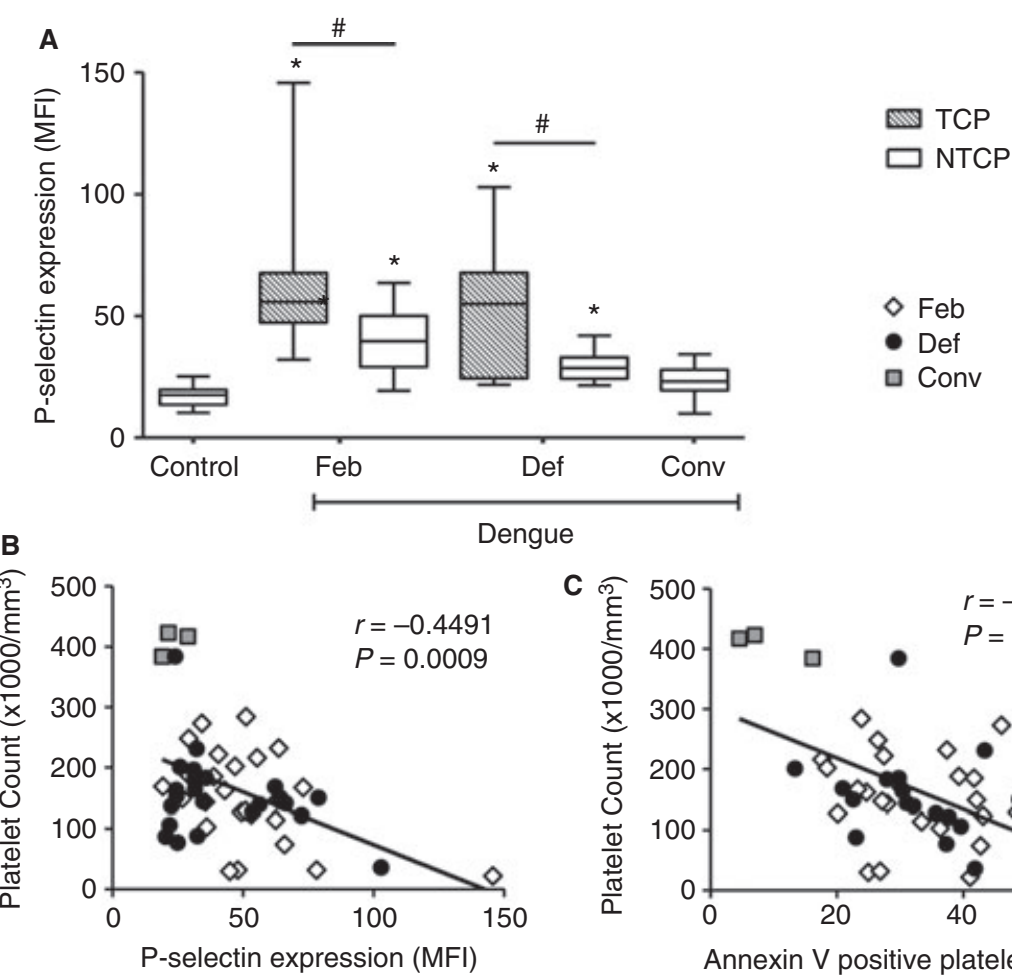

Def
$\square$ Conv

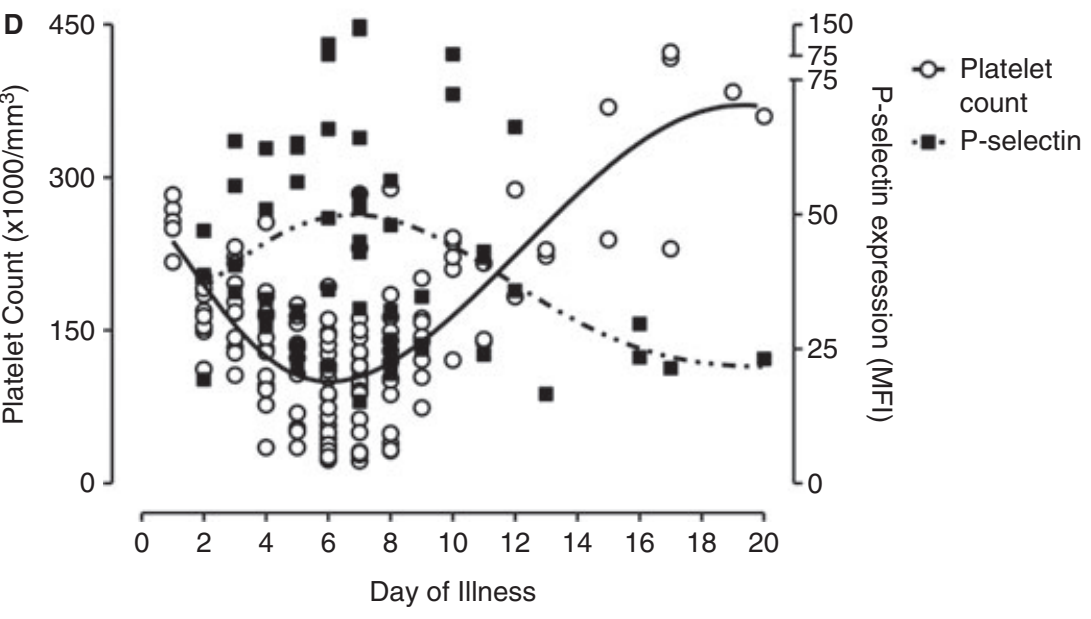

Fig. 2. Platelet counts in patients with dengue correlate with indices of platelet activation. (A) The mean fluorescence intensity (MFI) of Pselectin expression in thrombocytopenic (TCP) and non-thrombocytopenic (NTCP) dengue patients. Boxes indicate the median and interquartile ranges and whiskers indicate 5-95 percentile. (B-C) Platelet counts were plotted against the MFI of P-selectin expression (B) and the percentage of annexin V-binding platelets (C) in febrile (Feb) defervescence (Def) and convalescence (Conv) phases. In Panels B and C the analysis was restricted to platelet counts obtained on the same day that P-selectin and phosphatidylserine were analysed. (D) Platelet counts and the MFI of P-selectin expression were plotted against the day of illness in which each value was obtained. Non-linear regressions were traced according to the distribution of dots. ${ }^{*} P<0.05$ vs. control; $\# P<0.01$ TCP vs. NTCP.

or $32.6 \pm 13.7$ MFI for virus filtrate and mock retentate, respectively; $n=4, P<0.05$ ).

Phosphatidylserine exposure on platelets was also increased after $6 \mathrm{~h}$ of incubation with DENV-2 $(68.7 \pm 11.5 \%$ vs. $19.2 \pm 11.8 \%$ of positive platelets; $n=4, P=0.0052$ ) (Fig. 5C). To investigate the proportion of phosphatidylserine exposed as a result of platelet activation or apoptosis, platelets were incubated with DENV in presence of the pan-caspase inhibitor ZVAD- fmk. Blocking caspase activity did not affect P-selectin expression (Fig. 5D), but significantly $(P<0.05)$ impaired phosphatidylserine exposure on platelets (Fig. 5E). These data demonstrate that most of phosphatidylserine exposure depends on apoptosis pathways and only a small proportion depends on platelet activation.

In addition to infective DENV-2, we also exposed platelets to heat-inactivated virus $\left(56{ }^{\circ} \mathrm{C}, 1 \mathrm{~h}\right)$. Heat inactivation prevented DENV-2 from increasing P-selectin 

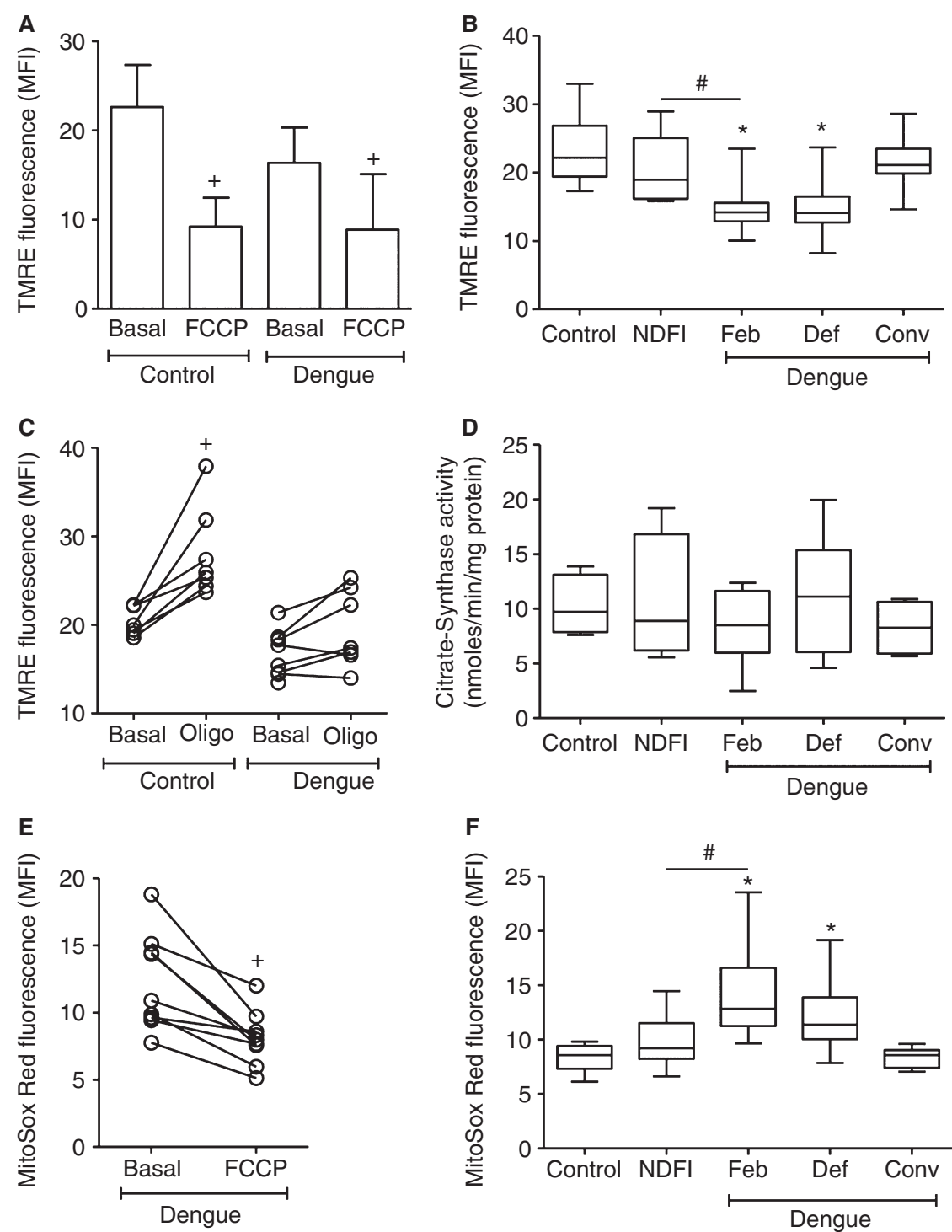

Fig. 3. Mitochondrial function is impaired in platelets from dengue-infected patients. (A-C) The mean fluorescence intensity (MFI) for TMRE, (D) the Citrate-Synthase activity, and (E-F) the MFI of MitoSoxRed in platelets from healthy subjects (control), patients with non-dengue febrile illness (NDFI), and dengue-infected patients in febrile (Feb), defervescence (Def), or convalescence (Conv) phases. (A, C and E) Mitochondrial responses in platelets treated with FCCP or oligomycin. Bars (A) depict the mean \pm stnadrd error of the mean (SEM) of 7-9 healthy participants and dengue patients; dots (C and E) represent tetramethylrhodamine ethyl ester (TMRE) or MitoSoxRed fluorescence in platelets from control or dengue patients before (basal) and after the treatments. Boxes (B, D and F) indicate median and interquartile ranges and whiskers indicate $5-95$ percentile. $+P<0.05$ vs. basal; $* P<0.05$ vs. control; $\# P<0.05$ vs. NDFI.

and phosphatidylserine on the surface of platelets (Fig. 5D,E).

DENV-2 also significantly impaired mitochondrial function in platelets as evidenced by a reduction in TMRE fluorescence (infectious DENV-2, $18.2 \pm 7.4$ MFI vs. $31.6 \pm 18.3 \mathrm{MFI}$ or $31.3 \pm 12.3 \mathrm{MFI}$ for heatinactivated and mock, respectively; $n=6, P<0.01)$ ) and increased MitoSOX Red fluorescence (infectious DENV$2,38.3 \pm 13.8$ MFI vs. $23.5 \pm 7.7$ MFI or $24.6 \pm 6.4$ MFI for heat inactivated-DENV-2 and mock, respectively; $n=6, P<0.01$ ) (Fig. 6A,B). Activated caspase-9 was also significantly higher in platelets exposed to DENV-2 (infectious DENV-2, $50.4 \pm 11.5$ MFI vs.
$28.6 \pm 12.3$ MFI or $29.0 \pm 5.6$ MFI for heat-inactivated DENV-2 and mock, respectively; $n=4, \quad P<0.01$ ) (Fig. 6C). Furthermore, a negative correlation was observed between $\Delta \Psi_{\mathrm{m}}$ and phosphatidylserine exposure (Fig. 6D). We observed that platelets with high phosphatidylserine exposure concomitantly exhibited reduced TMRE fluorescence (Fig. 6D insets).

\section{DENV-2 activates platelets through pathways that involve $D C-S I G N$}

Previous studies in other cell types have shown that DENV particles interact with surface molecules that include DC- 
A

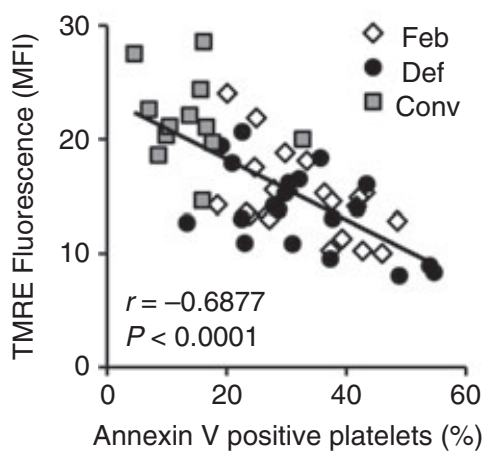

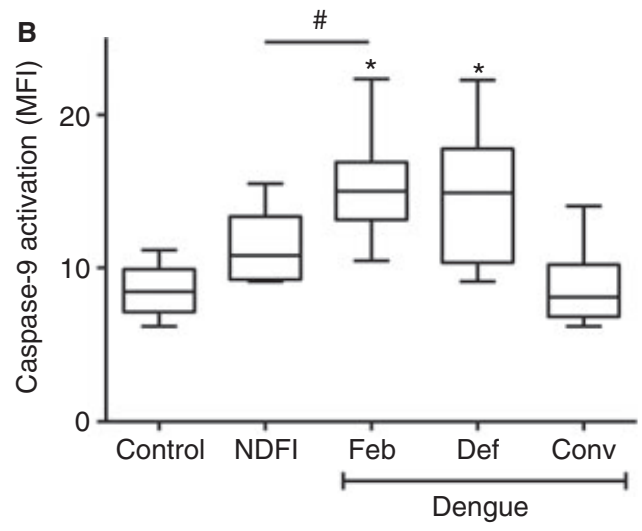

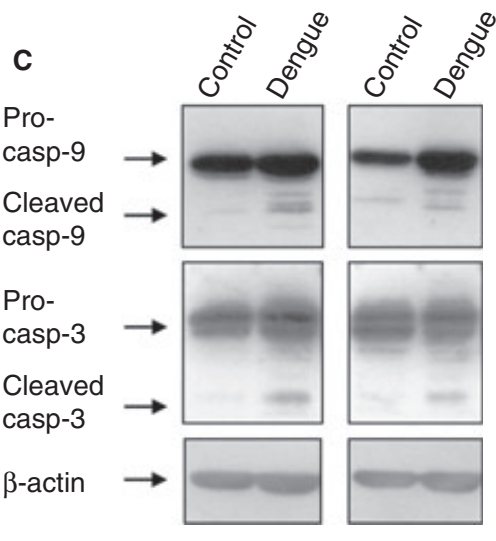

Fig. 4. Platelets apoptosis in patients with dengue. (A) The mean fluorescence intensity (MFI) for tetramethylrhodamine ethyl ester (TMRE) was plotted against the percentage of annexin V-binding platelets in febrile (Feb), defervescence (Def) and convalescence (Conv) dengue phases. (B) The MFI of caspase-9 activation in platelets freshly isolated from healthy subjects (control), patients with non-dengue febrile illness (NDFI) and dengue-infected patients in Feb, Def, and Conv. Boxes indicate median and interquartile ranges and whiskers indicate 5-95 percentile. (C) Western analysis of pro- and cleaved caspase-9 (casp-9) and caspase-3 (casp-3), and $\square$-actin in platelets isolated from control or dengue patients (representative of 5$)$. ${ }^{*} P<0.01$ vs. control; $\# P<0.05$ vs. NDFI.
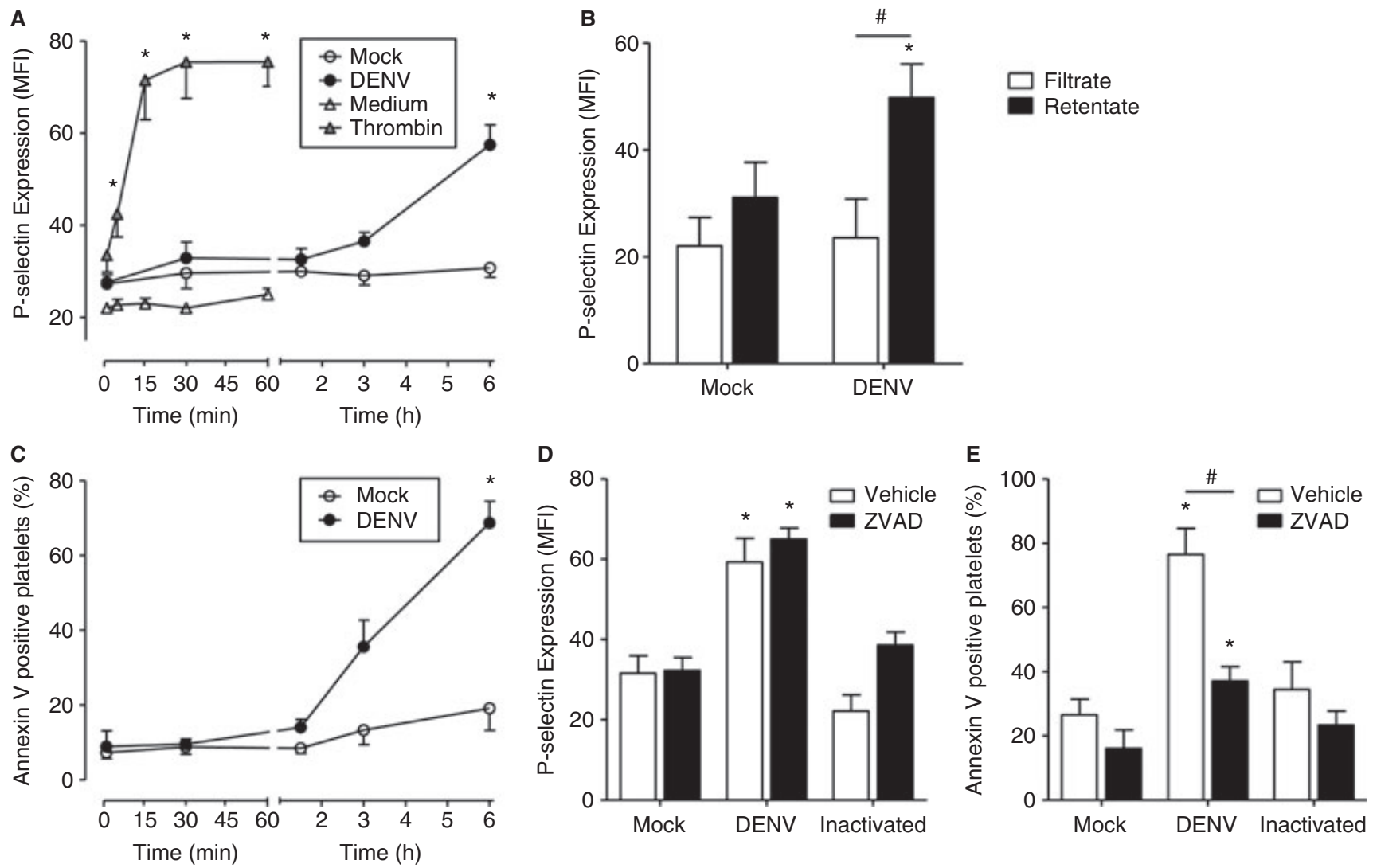

Fig. 5. DENV-2 induces platelet activation. The mean fluorescence intensity (MFI) of P-selectin expression (A, B and D) and the percentage $(\%)$ of annexin V-binding platelets (C and $\mathrm{E}$ ) in platelets exposed to DENV in vitro. (A and C) Platelets were exposed to mock or DENV-2 for $1,30 \mathrm{~min}, 1.5,3$ or $6 \mathrm{~h}$; or activated with thrombin for 1, 5, 15, 30 min or $1 \mathrm{~h}$. (B) Platelets were exposed (6 h) to the filtrate or the retentate of DENV-2 purified through centrifugation in a Centricon Filter, or mock processed in parallel. (D and E) Platelets were exposed (6 h) to mock, DENV or heat-inactivated DENV in the presence of the pan caspase inhibitor ZVAD-fmk. Dots and bars represent mean \pm standard error of the mean (SEM) of four independent experiments from individual donors. $* P<0.05$ vs. mock; $\# P<0.05$ vs. DENV filtrate or ZVAD-fmk treated platelets. 
A

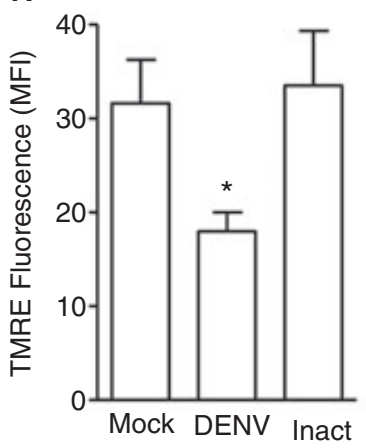

D

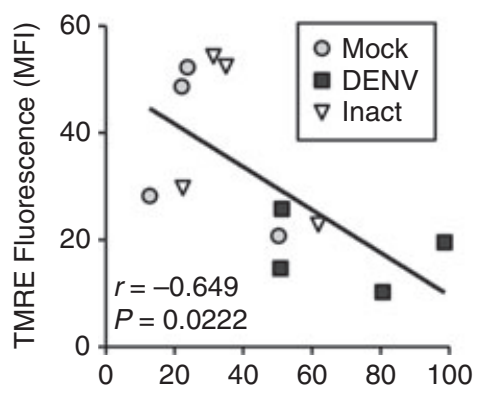

Annexin $\mathrm{V}$ positive platelets (\%)

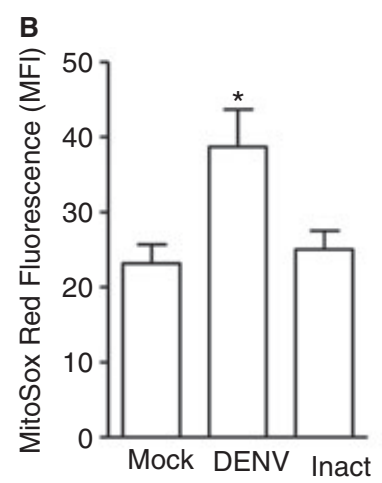

C
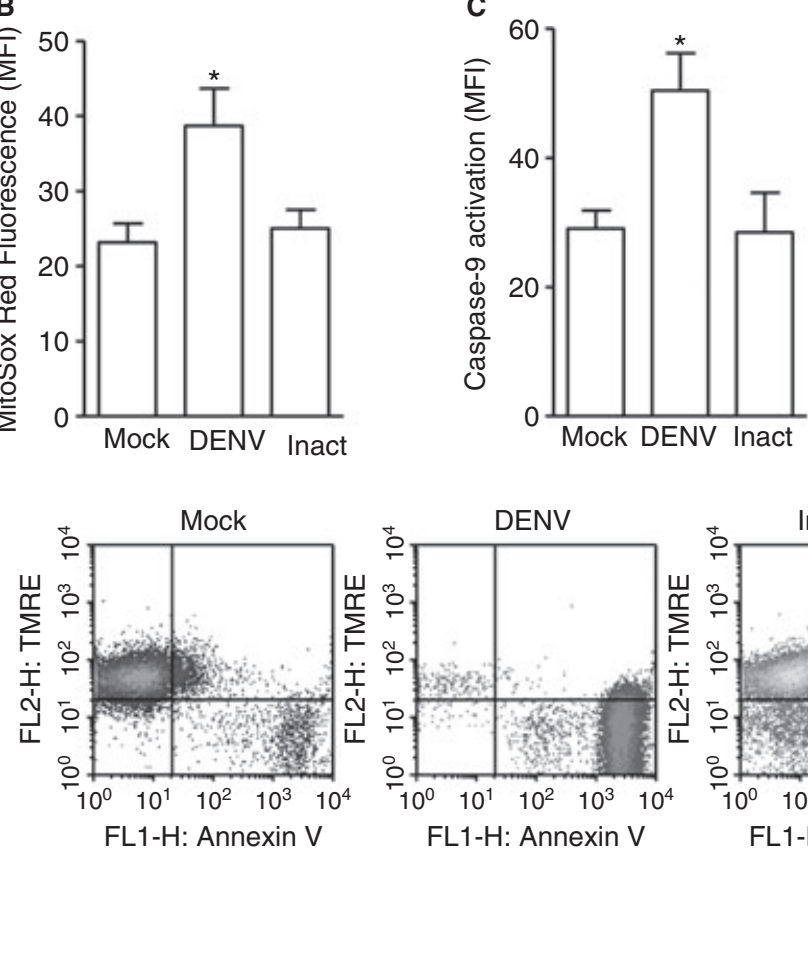

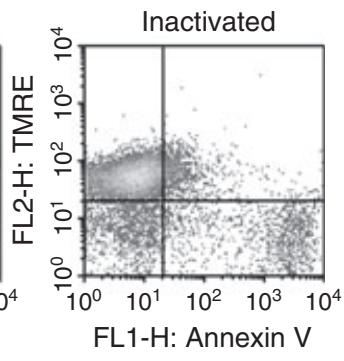

Fig. 6. DENV-2 induces mitochondrial dysfunction and apoptosis in platelets. The mean fluorescence intensity (MFI) for tetramethylrhodamine ethyl ester (TMRE) (A), MitoSoxRed (B) and caspase-9 activation (C) in platelets exposed for $6 \mathrm{~h}$ to mock, DENV-2 or heat-inactivated DENV2. The bars represent mean \pm standard error of the mean (SEM) of 4-6 independent experiments from individual donors. (D) The MFI for TMRE was plotted against the percentage of annexin V-binding platelets. Representative density plots are shown. $* P<0.01$ vs. mock.

SIGN and integrin $\alpha_{v} \beta_{3}[26,27]$. Although these receptors have been reported in platelets $[28,29]$, they have not been linked to DENV-induced platelet activation. To investigate their roles in platelet activation, platelets were incubated with neutralizing antibodies against DC-SIGN or $\alpha_{v}$ integrin subunit prior to exposure to DENV-2. Blocking of DC-SIGN, but not $\alpha_{v}$, prevented DENV-2 from increasing P-selectin expression on the surface of platelets (Fig. 7A). Decreased $\Delta \Psi_{\mathrm{m}}$ elicited by DENV-2 was similarly rescued by anti-DC-SIGN antibodies (Fig. 7B).

To clarify the role played by DC-SIGN-expressing platelets in DENV-induced platelet activation, we analyzed surface P-selectin expression in DC-SIGN-stained platelets gated as DC-SIGN positive or negative. DCSIGN positive platelets ranged from $13.4 \%$ to $37.5 \%$ $(21.4 \pm 8.5 \%)$ (Fig. 7C). DENV exposure did not affect DC-SIGN expression on platelets (Fig. 7D). As shown in Fig. 7E, DC-SIGN positive platelets exhibited increased P-selectin expression compared with DC-SIGN negative $(51.8 \pm 14.6 \mathrm{MFI}$ for DC-SIGN+ vs. $31.4 \pm 3.2 \mathrm{MFI}$ for DC-SIGN-; $n=4, P=0.0398)$.

\section{Discussion}

Thrombocytopenia is a common manifestation of dengue infection. Increased platelet clearance may occur in den- gue infection as consequence of platelet activation. However, the mechanisms underlying this remain elusive. Here, we show that platelets from dengue patients display classic signs of apoptosis that include increased phosphatidylserine exposure, mitochondrial depolarization and activation of caspase-9 and -3. Moreover, thrombocytopenia in patients with dengue strongly correlates with enhanced platelet activation and apoptosis. Our data also implicate DC-SIGN as a critical receptor in DENVdependent platelet activation.

Abnormal platelet function has been reported in dengue [30], but the underlying mechanisms are not fully understood. We consistently observed that platelets from DENV-infected patients were more activated compared with healthy subjects. Moreover, platelet activation was more pronounced in thrombocytopenic patients. This suggests that dengue may activate platelets and, as a result, facilitate platelet deposition in micro-vessels and/or clearance. Consistent with this hypothesis, increased phagocytosis of platelets by macrophages in patients with dengue was recently reported [6]. Sequestration of platelets in the liver and spleen of dengue-infected patients have also been observed [31].

Many factors influence platelet survival, which is reduced in patients with dengue [31]. One of these involves the intrinsic pathway of apoptosis [16]. It is 

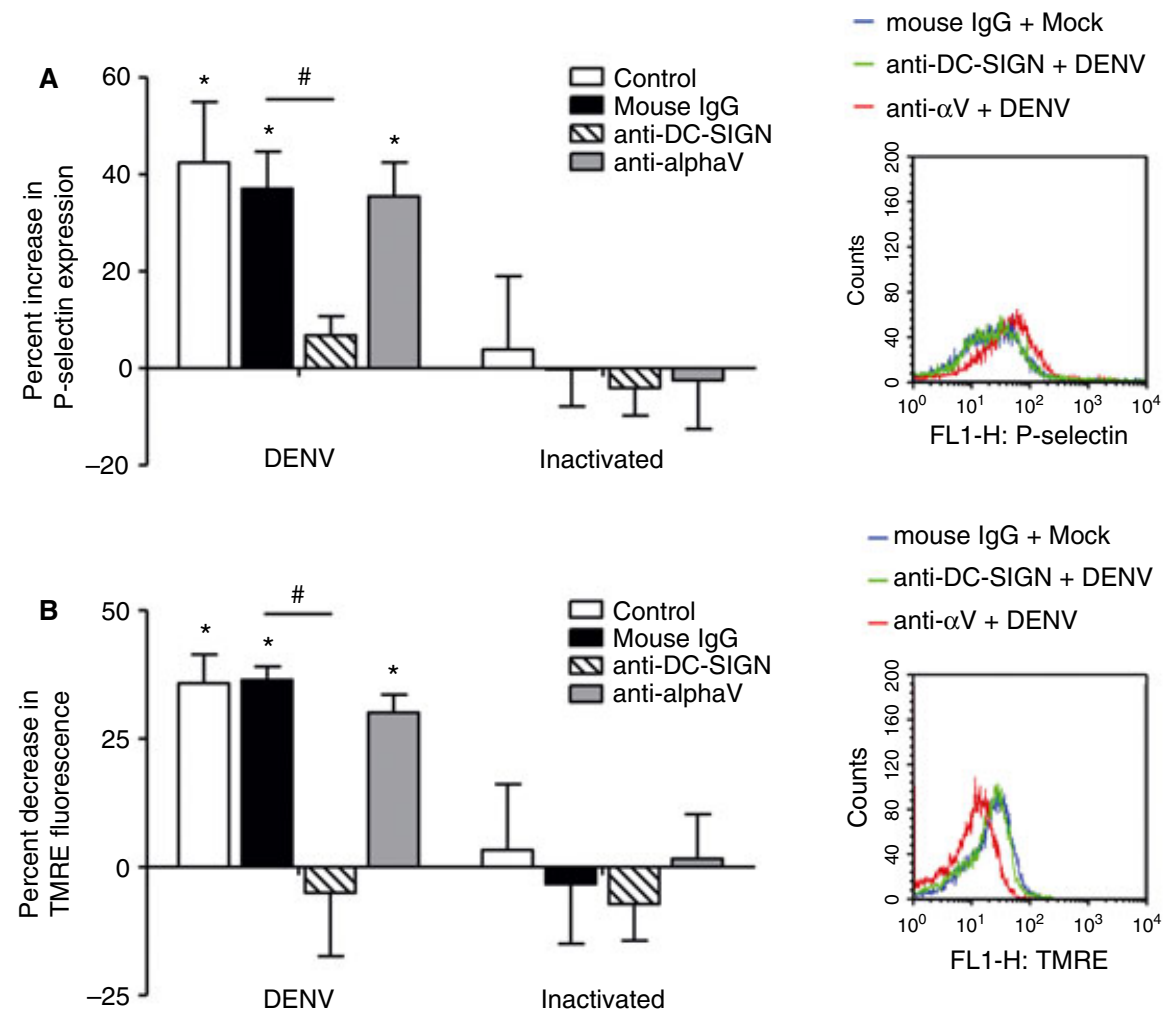

$$
\begin{aligned}
& \text { - mouse IgG + Mock } \\
& - \text { anti-DC-SIGN + DENV } \\
& - \text { anti- } \alpha \mathrm{V}+\text { DENV }
\end{aligned}
$$
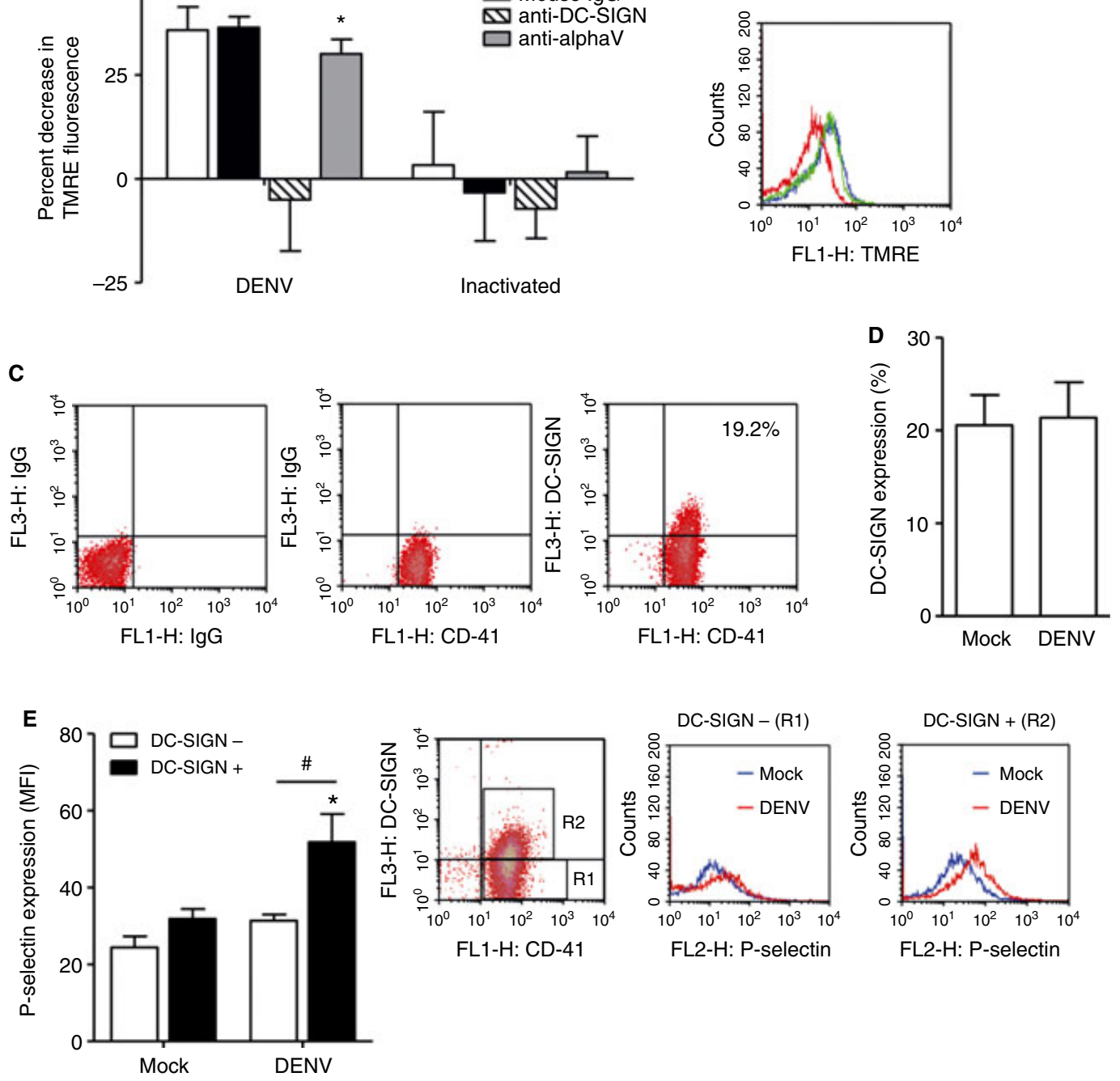

Fig. 7. DENV-2 activates platelets through mechanisms that involve DC-SIGN. Platelets were exposed for $6 \mathrm{~h}$ to mock, DENV-2 or heat-inactivated DENV-2 in the presence or absence of neutralizing antibodies against DC-SIGN or the $\alpha_{\mathrm{V}}$ integrin subunit. The percentage increase in P-selectin expression (A) or percentage decrease in tetramethylrhodamine ethyl ester (TMRE) fluorescence (B) related to mock values are shown. (C) Representative density plots showing the expression of DC-SIGN on platelets. (D) The percentage of DC-SIGN-expressing platelets exposed to mock or DENV. (E) Platelets exposed to mock or DENV-2 were stained for P-selectin and DC-SIGN. The mean fluorescence intensity (MFI) for P-selectin expression was assessed on platelets gated as DC-SIGN negative (DC-SIGN-) or positive (DC-SIGN+). Representative histograms and density plots are shown next to their corresponding graphs. Bars represent the mean \pm standard error of the mean (SEM) of four independent experiments from individual donors. ${ }^{*} P<0.05$ vs. mock; $\# P<0.05$ between anti-DC-SIGN or IgG isotype (A and B); or between DC-SIGN- and DC-SIGN+ (E). 
widely accepted that DENV induces apoptosis in host cells [32,33]. When DENV infects hepatocytes it increases mitochondrial permeability and, as a consequence, induces apoptosis [33]. We similarly observed mitochondrial dysfunction and apoptosis in platelets from patients with dengue. Moreover, DENV-induced phosphatidylserine exposure in vitro was largely dependent on caspase activation as assessed by ZVAD blockade, further supporting apoptotic-dependent mechanisms of cell death. Conceivably, mitochondrial dysfunction followed by activation of apoptosis pathways in platelets may contribute to the genesis of thrombocytopenia in dengue. Consistent with this possibility, we found that platelet counts in dengue-infected patients inversely correlate with phosphatidylserine exposure (Fig. 2C) and positively correlate with $\Delta \Psi_{\mathrm{m}}(r=0.4612, P<0.001)$ (data not shown).

It was previously reported that platelet activation and apoptosis followed distinct kinetics during dengue illness [34]. We similarly observed that phosphatidylserine exposure remained higher whereas $\mathrm{P}$-selectin presentation recovered to normal levels (data not shown). Platelet apoptosis has been shown to follow the platelet activation in vitro [35]. However, this was not the case in dengue infection, as P-selectin expression and phosphatidylserine exposure were concomitantly increased during the initial phase of infection. Furthermore, the kinetics of P-selectin expression and phosphatidylserine exposure were similar in platelets incubated with DENV in vitro (Fig. 5), suggesting that platelet apoptosis is directly induced by DENV. Other factors beyond DENV exposure may be responsible for platelet apoptosis in non-viremic phases. Although our results showed activation of the mitochondrial pathway of apoptosis, we cannot exclude participation of other cell death pathways as necrosis or apoptosis by extrinsic pathways.

In addition to mitochondrial dysfunction and activation of apoptosis pathways, we found that DENV-2 directly activated platelets isolated from healthy donors. Consistent with our observations, Ghosh et al. [12] previously demonstrated that DENV-2 induces morphological features of activation in platelets. Although these data indicate that the virus can directly activate platelets, we recognize that other factors can contribute to platelet activation including antibodies, cytokines, endothelial activation and coagulation factors among others [7]. It was previously shown that DENV-specific antibodies may potentiate DENV binding to platelets [36]. Thus, increased serum IgG levels in patients with secondary dengue may also contribute to platelet activation, even although we did not find significant differences in platelet activation and apoptosis from primary or secondary dengue infections (Table S2). Also, although in our study patients with severe dengue exhibited a trend towards increased platelet activation and apoptosis (data not shown), it was not possible to determine whether this correlates with more severe clinical outcome owing to the small size of our cohort.
The mechanisms by which dengue induces platelet activation are not known. Here we show that DC-SIGN supports DENV-induced P-selectin surface expression and mitochondrial dysfunction in platelets. Boukour et al, [28] previously demonstrated that platelets express DC-SIGN, a C-type lectin receptor that has demonstrated roles in DENV recognition in dendritic cells [26]. Blockade of DC-SIGN on the surface of platelets prevents DENV-2 from activating platelets and impairing $\Delta \Psi_{\mathrm{m}}$. Whether this is as a result of outside-in activation of DC-SIGN signaling pathways and/or internalization of DENV-2 into platelets requires further investigation. Regarding the latter, DC-SIGN facilitates entry of HIV into platelets [28] and DENV has been detected in platelets [13,37]. If DENV entry is necessary to induce platelet activation and apoptosis, other receptors such as FC $\gamma$ RIIa may be also involved, as it can mediate DENV entry in macrophages [38] and has demonstrated roles in Staphylococcus aureus-induced platelet activation [39].

In summary, we show that platelets from dengueinfected patients have impaired mitochondrial function and evidence of apoptosis, which probably contributes to the development of thrombocytopenia in dengue disease. As DENV can trigger mitochondrial dysfunction and platelet activation through mechanisms that involve DC-SIGN, therapeutic strategies that prevent DENV from interacting with DC-SIGN on platelets may have a role in the treatment of dengue-associated thrombocytopenia.

\section{Addendum}

E.D. Hottz, performed the majority of experiments, data analyzes and manuscript drafting and preparation; P.C.G. Nunes, performed virological and immunological analyses from patient plasma; R.M.R. Nogueira, experimental design and manuscript editing/review; M.F. Oliveira, experimental design and manuscript editing/review; R. Valls-de-Souza, patient enrolment and experimental analyzes; A.T. Da Poian, experimental design and manuscript editing/review; A.S. Weyrich, experimental design and manuscript editing/review; G.A. Zimmerman, experimental design and manuscript editing/review; P.T. Bozza and F.A. Bozza, directed all aspects of the study, data analyzes, and manuscript preparation and review.

\section{Acknowledgements}

We thank Gisele B. Lima for virus production, and Alan B. Carneiro, Luiz Felipe G. Souza, Edson F. Assis and Monique R. Q. Lima for technical assistance.

\section{Funding}

This work was supported by grants from Conselho Nacional de Desenvolvimento Científico e Tecnológico (CNPq), 
Fundação de Amparo a Pesquisa do Estado do Rio de Janeiro (FAPERJ), PAPES/FIOCRUZ, INBEB and PRONEX Dengue; and from the National Institutes of Health (HL066277, HL091754, and HL044525 awarded to A.S.W. and G.A.Z).

\section{Disclosure of Conflict of Interests}

The authors declare no competing financial interests.

\section{Supporting Information}

Additional Supporting Information may be found in the online version of this article:

Table S1. Characteristics of patients with non-dengue febrile illness (NDFI).

Table S2. Platelet activation, mitochondrial dysfunction, and apoptosis in platelets from patients with primary and secondary dengue infection.

\section{References}

1 Schmidt AC. Response to dengue fever-the good, the bad, and the ugly? $N$ Engl J Med 2010; 363: 484-7.

2 WHO. Dengue: guidelines for diagnosis, treatment, prevention and control. 2009.

3 Torrez EM. Dengue. Estudos Avanzados 2008; 22: 33-52.

4 Mourao MP, Lacerda MV, Macedo VO, Santos JB. Thrombocytopenia in patients with dengue virus infection in the Brazilian Amazon. Platelets 2007; 18: 605-12.

5 Schexneider KI, Reedy EA. Thrombocytopenia in dengue fever. Curr Hematol Rep 2005; 4: 145-8.

6 Honda S, Saito M, Dimaano EM, Morales PA, Alonzo MT, Suarez LA, Koike N, Inoue S, Kumatori A, Matias RR, Natividad FF, Oishi K. Increased phagocytosis of platelets from patients with secondary dengue virus infection by human macrophages. Am J Trop Med Hyg 2009; 80: 841-5.

7 Hottz E, Toley N, Zimmerman G, Weyrich A, Bozza F. Platelets in dengue infection. Drug Discov Today Dis Mech 2011; 8: e33-8.

8 La Russa VF, Innis BL. Mechanisms of dengue virus-induced bone marrow suppression. Baillieres Clin Haematol 1995; 8: 249-70.

9 Lin CF, Wan SW, Cheng HJ, Lei HY, Lin YS. Autoimmune pathogenesis in dengue virus infection. Viral Immunol 2006; 19: $127-32$.

10 Krishnamurti C, Peat RA, Cutting MA, Rothwell SW. Platelet adhesion to dengue-2 virus-infected endothelial cells. Am J Trop Med Hyg 2002; 66: 435-41.

11 Tsai JJ, Jen YH, Chang JS, Hsiao HM, Noisakran S, Perng GC. Frequency alterations in key innate immune cell components in the peripheral blood of dengue patients detected by FACS analysis. J Innate Immun 2011; 3: 530-40.

12 Ghosh K, Gangodkar S, Jain P, Shetty S, Ramjee S, Poddar P, Basu A. Imaging the interaction between dengue 2 virus and human blood platelets using atomic force and electron microscopy. J Electron Microsc 2008; 57: 113-8.

13 Noisakran S, Chokephaibulkit K, Songprakhon P, Onlamoon N, Hsiao HM, Villinger F, Ansari A, Perng GC. A re-evaluation of the mechanisms leading to dengue hemorrhagic fever. Ann $N Y$ Acad Sci 2009; 1171: E24-35.

14 Leytin V, Allen DJ, Mykhaylov S, Lyubimov E, Freedman J. Thrombin-triggered platelet apoptosis. J Thromb Haemost 2006; 4: $2656-63$.
15 Lopez JJ, Salido GM, Pariente JA, Rosado JA. Thrombin induces activation and translocation of Bid, Bax and Bak to the mitochondria in human platelets. J Thromb Haemost 2008; 6: 1780-8.

16 Mason KD, Carpinelli MR, Fletcher JI, Collinge JE, Hilton AA, Ellis S, Kelly PN, Ekert PG, Metcalf D, Roberts AW, Huang DC, Kile BT. Programmed anuclear cell death delimits platelet life span. Cell 2007; 128: 1173-86.

17 Falconar AK, De Plata E, Romero-Vivas CM. Altered enzymelinked immunosorbent assay immunoglobulin $\mathrm{M}(\operatorname{IgM}) / \operatorname{IgG}$ optical density ratios can correctly classify all primary or secondary dengue virus infections 1 day after the onset of symptoms, when all of the viruses can be isolated. Clin Vaccine Immunol 2006; 13: 1044-51.

18 Lanciotti RS, Calisher CH, Gubler DJ, Chang GJ, Vorndam AV. Rapid detection and typing of dengue viruses from clinical samples by using reverse transcriptase-polymerase chain reaction. J Clin Microbiol 1992; 30: 545-51.

19 Johnson BW, Russell BJ, Lanciotti RS. Serotype-specific detection of dengue viruses in a fourplex real-time reverse transcriptase PCR assay. J Clin Microbiol 2005; 43: 4977-83.

20 Weyrich AS, Elstad MR, McEver RP, McIntyre TM, Moore KL, Morrissey JH, Prescott SM, Zimmerman GA. Activated platelets signal chemokine synthesis by human monocytes. $J$ Clin Invest 1996; 97: 1525-34.

21 Alves LR, Costa ES, Sorgine MH, Nascimento-Silva MC, Teodosio C, Barcena P, Castro-Faria-Neto HC, Bozza PT, Orfao A, Oliveira PL, Maya-Monteiro CM. Heme-oxygenases during erythropoiesis in K562 and human bone marrow cells. PLoS ONE 2011; 6: e21358.

22 Conceicao TM, El-Bacha T, Villas-Boas CS, Coello G, Ramirez J, Montero-Lomeli M, Da Poian AT. Gene expression analysis during dengue virus infection in HepG2 cells reveals virus control of innate immune response. J Infect 2010; 60: 65-75.

23 Estaquier J, Vallette F, Vayssiere JL, Mignotte B. The mitochondrial pathways of apoptosis. Adv Exp Med Biol 2012; 942: 157-83.

24 Jobe SM, Wilson KM, Leo L, Raimondi A, Molkentin JD, Lentz SR, Di Paola J. Critical role for the mitochondrial permeability transition pore and cyclophilin $\mathrm{D}$ in platelet activation and thrombosis. Blood 2008; 111: 1257-65.

25 Verhoeven AJ, Verhaar R, Gouwerok EG, De Korte D. The mitochondrial membrane potential in human platelets: a sensitive parameter for platelet quality. Transfusion 2005; 45: 82-9.

26 Tassaneetrithep B, Burgess TH, Granelli-Piperno A, Trumpfheller C, Finke J, Sun W, Eller MA, Pattanapanyasat K, Sarasombath S, Birx DL, Steinman RM, Schlesinger S, Marovich MA. DC-SIGN (CD209) mediates dengue virus infection of human dendritic cells. $J$ Exp Med 2003; 197: 823-9.

27 Zhang JL, Wang JL, Gao N, Chen ZT, Tian YP, An J. Up-regulated expression of beta3 integrin induced by dengue virus serotype 2 infection associated with virus entry into human dermal microvascular endothelial cells. Biochem Biophys Res Commun 2007; 356: 763-8.

28 Boukour S, Masse JM, Benit L, Dubart-Kupperschmitt A, Cramer EM. Lentivirus degradation and DC-SIGN expression by human platelets and megakaryocytes. J Thromb Haemost 2006; 4: 426-35.

29 Paul BZ, Vilaire G, Kunapuli SP, Bennett JS. Concurrent signaling from Galphaq- and Galphai-coupled pathways is essential for agonist-induced alphavbeta3 activation on human platelets. $J$ Thromb Haemost 2003; 1: 814-20.

30 Srichaikul T, Nimmannitya S. Haematology in dengue and dengue haemorrhagic fever. Baillieres Best Pract Res Clin Haematol 2000; 13: 261-76.

31 Mitrakul C, Poshyachinda M, Futrakul P, Sangkawibha N, Ahandrik S. Hemostatic and platelet kinetic studies in dengue hemorrhagic fever. Am J Trop Med Hyg 1977; 26: 975-84. 
32 Courageot MP, Catteau A, Despres P. Mechanisms of dengue virus-induced cell death. Adv Virus Res 2003; 60: 157-86.

33 El-Bacha T, Midlej V, Pereira da Silva AP, Silva da Costa L, Benchimol M, Galina A, Da Poian AT. Mitochondrial and bioenergetic dysfunction in human hepatic cells infected with dengue 2 virus. Biochim Biophys Acta 2007; 1772: 1158-66.

34 Alonzo MT, Lacuesta TL, Dimaano EM, Kurosu T, Suarez LA, Mapua CA, Akeda Y, Matias RR, Kuter DJ, Nagata S, Natividad FF, Oishi K. Platelet apoptosis and apoptotic platelet clearance by macrophages in secondary dengue virus infections. $J$ Infect Dis 2012; 205: 1321-9.

35 Leytin V, Allen DJ, Mutlu A, Mykhaylov S, Lyubimov E, Freedman J. Platelet activation and apoptosis are different phenomena: evidence from the sequential dynamics and the magnitude of responses during platelet storage. Br J Haematol 2008; 142: 494-7.
36 Wang S, He R, Patarapotikul J, Innis BL, Anderson R. Antibody-enhanced binding of dengue-2 virus to human platelets. Virology 1995; 213: 254-7.

37 Noisakran S, Gibbons RV, Songprakhon P, Jairungsri A, Ajariyakhajorn C, Nisalak A, Jarman RG, Malasit P, Chokephaibulkit K, Perng GC. Detection of dengue virus in platelets isolated from dengue patients. Southeast Asian J Trop Med Public Health 2009; 40: 253-62.

38 Wahala WM, Silva AM. The human antibody response to dengue virus infection. Viruses 2011; 3: 2374-95.

39 Fitzgerald JR, Loughman A, Keane F, Brennan M, Knobel M, Higgins J, Visai L, Speziale P, Cox D, Foster TJ. Fibronectinbinding proteins of Staphylococcus aureus mediate activation of human platelets via fibrinogen and fibronectin bridges to integrin GPIIb/IIIa and IgG binding to the FcgammaRIIa receptor. Mol Microbiol 2006; 59: 212-30. 